\title{
Repetitive strain injury in a data processing department of a bank
}

\author{
* Núcleo de Investigações em Trabalho e Saúde do Instituto de Saúde \\ **Centro de Referência em Saúde do Trabalhador da Secretaria de Estado da \\ Saúde de São Paulo
}

The objective of the present study was to verify the prevalence of Repetitive Strain Injury (RSI) diagnosis in a data processing department of a company in the banking business. This company employed 2,898 people, and the study was conducted by a surveillance process.

Through inspection procedures and meetings with representatives of the workers and from the company, a hypothesis was formed that certain offices (1,572 people) within the company had a higher prevalence of RSI than the other offices $(1,416)$.

To test this hypothesis, a questionnaire for self-reported complaints was set up. This questionnaire included: non-specific data about the subjects personal labor history, osteomuscular complaints, changes in everyday habits due to symptoms, a mental health screening (SRQ-20), visual complaints and the use of medication.

This questionnaire was applied randomly in the sample at risk that was divided by gender and age (group 1). An ageand gender-matched control group that included the remaining people from the same offices was used for comparison (group 2).

In the population studied:

$63.9 \%$ were males and $36.1 \%$ were females.

Age distribution was:

$-3.4 \%$ of the sample: 18 to 20 years old;

$-38.3 \%$ of the sample: 12 to 30 years old;

$-41.9 \%$ of the sample: 31 to 40 years old;

$-14.1 \%$ of the sample: 41 to 50 years old;

$-1.0 \%$ of the sample: 51 years old or older.

Comparing groups 1 and 2, a predominance of subjects (37\%) with a high school diploma was evident in group 1, whereas in group 2 , a predominance of subjects (64.4\%) with a college degree was recorded.

As for the group 1 and 2 positions, it was observed that:

- services operator/clearing clerk and data entry personnel were positions exclusive to group 1

- scribe/administrative assistant, representative operator, service technician, operational service technician (junior and senior), manager/service director/service manager/super- intendent were positions that predominated in group 1 .

- Engineer, computer technician and systems manager/ systems coordinator were positions exclusive to group 2 .

- Secretary, analyst, programmer and computer production analyst were positions that predominated in group 2 .

The data above do not represent all the positions that exist in that data processing department. It shows, however, the positions that comprised the sample are the main positions existing in that data processing department.

There is no significant difference between the average time length in the position for group 1 (6.84 years) and 2 (6.96 years).A significantly larger number of subjects from group 1 had a previous position within the same data processing department $(\mathrm{p}<0.05)$. However, in group 1 , there are no significant differences indicating that these former positions required any type of repetitive movements. Likewise, no significant association was recorded regarding a previous position elsewhere in another company where repetitive movements were required (16.3\% for group 1 and $20.0 \%$ for group 2 ). No statistically significant correlation was found for the item regarding working at a second job (group 1 gave a $4.8 \%$ positive answer, and $2.6 \%$ in group 2 ). Likewise, no statistically significant correlation was recorded for the item regarding repetitive movements in this second job. A significant higher frequency $(\mathrm{p}<0.001)$ of positive answers in group 1 was recorded for working overtime $(43.4 \%$ of the sample in group 1 worked overtime as opposed to $23.5 \%$ in group 2 ). A significant difference $(\mathrm{p}<0.05)$ was found in the self-image of their professional activity requiring repetitive movements (67.4\% and $60.4 \%$ for groups 1 and 2, respectively).

A significant correlation was not recorded regarding other activities outside the workplace requiring repetitive movements for groups 1 and 2 (26.4\% and $26.9 \%$ of the subjects in group 1 and 2, respectively, exerted repetitive movements outside the workplace).

As for the osteo-muscular complaints, the authors found a significantly higher frequency of responses nearly every day or once in a while in group 1. Pain sites and complaint types were:

- pain in the legs $(\mathrm{p}<0.001)$; 
— pain and fatigue/heaviness in the arms $(\mathrm{p}<0.001)$;

- pain and stiffness in the wrists $(\mathrm{p}<0.001)$;

- tingling/numbness in the wrists $(\mathrm{p}<0.05)$;

- tiredness/heaviness in the hands $(\mathrm{p}<0.001)$;

- pain, stiffness, lack of strength and tingling/numb-

ness in the hands $(\mathrm{p}<0.05)$. The significantly more frequent complaint for group 2 was neck stiffness ( $<<0.05$ ).

The answer nearly every day alone showed statistically higher frequencies for group 1 for the following complaints:

— back pain $(\mathrm{p}<0.001)$;

— pain in the legs $(\mathrm{p}<0.001)$;

— pain in the arms $(\mathrm{p}<0.05)$;

— stiffness in the wrists $(\mathrm{p}<0.001)$;

- tiredness and lack of strength in the hands.

No statistically higher frequencies were recorded for group 2 .

No statistically higher frequencies were found for:

— neck pain;

— stiffness in the back;

— tiredness/weight and tingling/numbness in the legs;

— pain $(\mathrm{p}<0.05)$, tiredness/weight, tingling/numbness in the shoulders;

— tingling/numbness in the arms.

The statistical analyses demonstrate a significant correlation between belonging to group 1 and time off for medical treatment $(\mathrm{p}<0.05)$ or a visit to an emergency room due to the complaints. Likewise, the statistical analysis demonstrates a significant correlation for the use of pain killers $(p<0.05$ significant for nearly every day and once in a while responses).
When the authors compare the nearly every day type of response with the other responses, a statistically significant difference is found for use of sedative/tranquilizer medication as well as complaints about problems with concentration ( $\mathrm{p}<0.05$ for both items).

No statistically significant difference was found for both groups regarding the SRQ-20. Other correlations should be further investigated regarding this item.

In summary, in group 1 the repetitive activities predominates as confirmed by means of survey procedures at the data processing office. In the comparision performed in this investigation, the authors recorded that the personnel from the offices considered at risk for RSI were transferred from offices within the data processing department. These subjects declared overtime more frequently, a self-awareness of about their work-related activity requiring more repetitive movements than the control group. Several osteo-muscular complaints were mentioned by group 1 subjects. These complaints were statistically more frequent than in the control group. This indicates a larger body of adverse consequences to the health in group 1 subjects as opposed to the control group. Group 1 subjects recorded more often time off for medical treatment, more frequent visits to an emergency room because of pain and other symptoms. Analgesic medication use on a nearly everyday basis or once in a while was also statistically more frequent in group 1 than in the control group. When the nearly every day frequency is considered alone, the information about troubles with concentration or use of tranquilizing medication shows a statistically significant difference for group 1 . 